\title{
Riccati and Ermakov Equations in Time-Dependent and Time-Independent Quantum Systems ${ }^{\star}$
}

\author{
Dieter SCHUCH
}

Institut für Theoretische Physik, J.W. Goethe-Universität, Max-von-Laue-Str. 1, D-60438 Frankfurt am Main, Germany

E-mail:schuch@em.uni-frankfurt.de

Received December 28, 2007, in final form May 07, 2008; Published online May 12, 2008

Original article is available at http://www.emis.de/journals/SIGMA/2008/043/

\begin{abstract}
The time-evolution of the maximum and the width of exact analytic wave packet (WP) solutions of the time-dependent Schrödinger equation (SE) represents the particle and wave aspects, respectively, of the quantum system. The dynamics of the maximum, located at the mean value of position, is governed by the Newtonian equation of the corresponding classical problem. The width, which is directly proportional to the position uncertainty, obeys a complex nonlinear Riccati equation which can be transformed into a real nonlinear Ermakov equation. The coupled pair of these equations yields a dynamical invariant which plays a key role in our investigation. It can be expressed in terms of a complex variable that linearizes the Riccati equation. This variable also provides the time-dependent parameters that characterize the Green's function, or Feynman kernel, of the corresponding problem. From there, also the relation between the classical and quantum dynamics of the systems can be obtained. Furthermore, the close connection between the Ermakov invariant and the Wigner function will be shown. Factorization of the dynamical invariant allows for comparison with creation/annihilation operators and supersymmetry where the partner potentials fulfil (real) Riccati equations. This provides the link to a nonlinear formulation of timeindependent quantum mechanics in terms of an Ermakov equation for the amplitude of the stationary state wave functions combined with a conservation law. Comparison with SUSY and the time-dependent problems concludes our analysis.
\end{abstract}

Key words: Riccati equation; Ermakov invariant; wave packet dynamics; nonlinear quantum mechanics

2000 Mathematics Subject Classification: 37J15; 81Q05; 81Q60; 81S30

\section{Introduction}

Participating in a conference on nonlinear mathematical physics taking place in Kiev, one can find more colleagues than usual who are familiar with the name Ermakov; some are even able to tell where one can find traces of him from the time he used to work at the university in Kiev. This period was, however, before quantum mechanics was established and Vasili Petrovich Ermakov (1845-1922) died already four years before Schrödinger published his celebrated equation [1]. So, what can be his relation with quantum theory? The second name in the title of this paper also seems to be surprising in this context because Jacopo Riccati (1676-1754) was born actually 250 years prior to Schrödinger's publication! The surprising answer to our question is that Ermakov's and Riccati's contributions are not only closely connected with quantum theory in the form established by Schrödinger and Heisenberg, but also with later formulations due to Feynman and Wigner. Therefore, their work plays a central role in finding underlying connections amongst all these different formulations.

\footnotetext{
${ }^{\star}$ This paper is a contribution to the Proceedings of the Seventh International Conference "Symmetry in Nonlinear Mathematical Physics" (June 24-30, 2007, Kyiv, Ukraine). The full collection is available at http://www.emis.de/journals/SIGMA/symmetry2007.html
} 
One way of showing how Riccati and Ermakov enter the formalism of quantum theory is the study of cases where exact analytic Gaussian wave packet (WP) solutions of the time-dependent Schrödinger equation (SE) exist, in particular, the harmonic oscillator (HO) and the free motion. Therefore, in the following, we will restrict the discussion of the time-dependent problems to the (one-dimensional) HO (where the frequency $\omega$ may also be time-dependent) and the free motion is obtained in the limit $\omega \rightarrow 0$. In Section 2, it will be shown how a (complex) nonlinear Riccati equation that governs the dynamics of a typical quantum effect, namely, the position uncertainty, emerges from the WP solution of the time-dependent SE. This Riccati equation can be transformed into a (real) nonlinear Ermakov equation that, together with the classical Newtonian equation of the system, provides a corresponding dynamical invariant, the so-called Ermakov invariant, that will play an important role in our discussion. As shown in Section 3, the linearization of the Riccati equation can be accomplished by introducing new complex variables. These variables are connected with the time-dependent parameters that characterize the time-dependent Green's function, also called Feynman kernel, of the corresponding problem. They also provide the key to understanding the relations between the dynamics of the classical and quantum aspects of the system; in particular, the importance of the initial position uncertainty will become obvious. Furthermore, in Section 4, the close relationship between the Ermakov invariant and the Wigner function will be shown. The link to the occurrence of Riccati and Ermakov equations in time-independent quantum mechanics is obtained in Section 5. Factorization of the Ermakov invariant allows for a comparison with creation and annihilation operators as well as their generalizations used in supersymmetry (SUSY). In the latter case, the supersymmetric partner potentials fulfil real Riccati equations. This shows similarities with a nonlinear formulation of time-independent quantum mechanics by Reinisch [2], where he obtains an Ermakov equation (with spatial derivatives instead of temporal ones) for the amplitude of the stationary state wave functions. This Ermakov equation is related with a kind of complexification of the Riccati equation appearing in SUSY. Finally, in Section 6, the main results will be summarized and possible generalizations mentioned.

\section{Dynamics of Gaussian wave packets and Ermakov invariant}

Starting point of our investigation is the time-dependent SE for the HO with frequency $\omega(t)$,

$$
i \hbar \frac{\partial}{\partial t} \Psi_{\mathrm{WP}}=\left\{-\frac{\hbar^{2}}{2 m} \frac{\partial^{2}}{\partial x^{2}}+\frac{m}{2} \omega^{2}(t) x^{2}\right\} \Psi_{\mathrm{WP}}
$$

that possesses Gaussian WP solutions of the type

$$
\Psi_{\mathrm{WP}}(x, t)=N(t) \exp \left\{i\left[y(t) \tilde{x}^{2}+\frac{\langle p\rangle}{\hbar} \tilde{x}+K(t)\right]\right\},
$$

where $y(t)=y_{\mathrm{R}}(t)+i y_{\mathrm{I}}(t), \tilde{x}=x-\langle x\rangle=x-\eta(t)$ (i.e., $\langle x\rangle=\eta(t)$ is the classical trajectory calculated as mean value $\left.\int \Psi_{\mathrm{WP}}^{*} x \Psi_{\mathrm{WP}} d x=\langle x\rangle\right),\langle p\rangle=m \dot{\eta}$ is the classical momentum and $N(t)$ and $K(t)$ are purely time-dependent terms that are not relevant for the following discussion.

Inserting the WP (2) into equation (1) yields the equations of motion for $\eta(t)$ and $y(t)$. The equation for the WP maximum, located at $x=\eta(t)$, is just the classical equation of motion

$$
\ddot{\eta}+\omega^{2}(t) \eta=0,
$$

where overdots denote time derivatives.

The equation of motion for the complex quantity $y(t)$ that is connected with the WP width and, thus, the position uncertainty, is given by the complex Riccati-type equation

$$
\frac{2 \hbar}{m} \dot{y}+\left(\frac{2 \hbar}{m} y\right)^{2}+\omega^{2}(t)=0 .
$$


Equation (4) can be separated into real and imaginary parts,

$$
\begin{aligned}
& \operatorname{Im}: \frac{2 \hbar}{m} \dot{y}_{\mathrm{I}}+2\left(\frac{2 \hbar}{m} y_{\mathrm{I}}\right)\left(\frac{2 \hbar}{m} y_{\mathrm{R}}\right)=0 \\
& \operatorname{Re}: \frac{2 \hbar}{m} \dot{y}_{\mathrm{R}}+\left(\frac{2 \hbar}{m} y_{\mathrm{R}}\right)^{2}-\left(\frac{2 \hbar}{m} y_{\mathrm{I}}\right)^{2}+\omega^{2}(t)=0 .
\end{aligned}
$$

The real part $y_{\mathrm{R}}(t)$ can be eliminated from equation (6) by solving equation (5) for $y_{\mathrm{R}}$ and inserting the result into equation (6).

It is useful to introduce a new variable $\alpha(t)$ that is connected with $y_{\mathrm{I}}(t)$ via

$$
\frac{2 \hbar}{m} y_{\mathrm{I}}=\frac{1}{\alpha^{2}(t)}
$$

where $\alpha(t)$ is directly proportional to the WP width, or position uncertainty, i.e., $\alpha=\left(\frac{m}{2 \hbar y_{\mathrm{I}}}\right)^{1 / 2}=$ $\left(\frac{2 m\left\langle\tilde{x}^{2}(t)\right\rangle}{\hbar}\right)^{1 / 2}$ with $\left\langle\tilde{x}^{2}\right\rangle=\left\langle x^{2}\right\rangle-\langle x\rangle^{2}$. Inserting this definition (7) into equation (5) shows that the real part of $y(t)$ just describes the relative change in time of the WP width,

$$
\frac{2 \hbar}{m} y_{\mathrm{R}}=\frac{\dot{\alpha}}{\alpha}=\frac{1}{2} \frac{\frac{d}{d t}\left\langle\tilde{x}^{2}\right\rangle}{\left\langle\tilde{x}^{2}\right\rangle} .
$$

Together with definition (7), this finally turns equation (6) into

$$
\ddot{\alpha}+\omega^{2}(t) \alpha=\frac{1}{\alpha^{3}},
$$

the so-called Ermakov equation.

It has been shown by Ermakov in 1880 [3] that the system of differential equations (3) and (9), coupled via the possibly time-dependent frequency $\omega$, leads to a dynamical invariant that has been rediscovered by several authors in the 20th century [4],

$$
I_{\mathrm{L}}=\frac{1}{2}\left[(\dot{\eta} \alpha-\eta \dot{\alpha})^{2}+\left(\frac{\eta}{\alpha}\right)^{2}\right]=\text { const. }
$$

It is straightforward to show that $\frac{d}{d t} I_{\mathrm{L}}=0$; a proof following Ermakov's method can be found in [5]. The Ermakov invariant not only depends on the classical variables $\eta(t)$ and $\dot{\eta}(t)$, but also on the quantum uncertainty connected with $\alpha(t)$ and $\dot{\alpha}(t)$. Additional interesting insight into the relation between the variables $\eta$ and $\alpha$ can be obtained from a different treatment of the Riccati equation (4).

\section{Linearization of the complex Riccati equation, Feynman kernel and quantum-classical connection}

Introducing a new complex variable $\lambda(t)$, the complex variable in the Riccati equation (4) can be replaced by the logarithmic time-derivative of $\lambda$, i.e.,

$$
\left(\frac{2 \hbar}{m} y\right)=\frac{\dot{\lambda}}{\lambda},
$$

thus turning the nonlinear Riccati equation into the complex linear equation

$$
\ddot{\lambda}+\omega^{2}(t) \lambda=0,
$$

looking exactly like equation (3) for $\eta(t)$, which is not just by accident, as will be shown later. 
The complex variable $\lambda(t)$ can be written in polar coordinates as well as in cartesian coordinates, i.e.,

$$
\lambda=\alpha e^{i \varphi}=u+i z .
$$

The choice of the symbol $\alpha$ for the absolute value of $\lambda$ is also not coincidental, as will now be shown.

\subsection{Polar form of the complex linearization variable}

Writing relation (11) in polar coordinates yields

$$
\frac{\dot{\lambda}}{\lambda}=\frac{\dot{\alpha}}{\alpha}+i \dot{\varphi}=\left(\frac{2 \hbar}{m} y\right)
$$

The real part already looks identical to the one given in equation (8). So, the absolute value of $\lambda$ would be (up to a constant factor) identical with the square root of the position uncertainty $\left\langle\tilde{x}^{2}\right\rangle$, if

$$
\dot{\varphi}=\frac{1}{\alpha^{2}}
$$

is fulfilled. This can easily be verified by inserting $\left(\frac{2 \hbar}{m} y_{\mathrm{R}}\right)$ and $\left(\frac{2 \hbar}{m} y_{\mathrm{I}}\right)$, as given in equation (12), into the imaginary part of the Riccati equation, thus turning equation (5) into

$$
\ddot{\varphi}+2 \frac{\dot{\alpha}}{\alpha} \dot{\varphi}=0
$$

in agreement with equation (13). From equation (6) for the real part then, again, the Ermakov equation is obtained as

$$
\ddot{\alpha}+\omega^{2}(t) \alpha=\alpha \dot{\varphi}^{2}=\frac{1}{\alpha^{3}} .
$$

\subsection{Cartesian form and Feynman kernel}

After the physical meaning of the absolute value of $\lambda$ in polar coordinates and its relation to the phase angle via (13) have been clarified, the interpretation of the cartesian coordinates $u$ and $z$ needs to be ascertained. For this purpose, it can be utilized that the WP solution (2) at time $t$ can also be obtained with the help of an initial WP at, e.g., $t^{\prime}=0$ and a time-dependent Green's function, also called time-propagator or Feynman kernel, via

$$
\Psi_{\mathrm{WP}}(x, t)=\int d x^{\prime} G\left(x, x^{\prime}, t, t^{\prime}=0\right) \Psi_{\mathrm{WP}}\left(x^{\prime}, 0\right) .
$$

For the considered Gaussian WP with initial distribution

$$
\Psi_{\mathrm{WP}}\left(x^{\prime}, 0\right)=\left(\frac{m \beta_{0}}{\pi \hbar}\right)^{1 / 4} \exp \left\{\frac{i m}{2 \hbar}\left[i \beta_{0} x^{\prime 2}+2 \frac{p_{0}}{m} x^{\prime}\right]\right\},
$$

where $\beta_{0}=\frac{\hbar}{2 m\left\langle\tilde{x}^{2}\right\rangle_{0}}=\frac{1}{\alpha_{0}^{2}}$ and $p_{0}=\langle p\rangle(t=0)$, the Green's function can be written as

$$
G\left(x, x^{\prime}, t, 0\right)=\left(\frac{m}{2 \pi i \hbar \alpha_{0} z}\right)^{1 / 2} \exp \left\{\frac{i m}{2 \hbar}\left[\frac{\dot{z}}{z} x^{2}-2 \frac{x}{z}\left(\frac{x^{\prime}}{\alpha_{0}}\right)+\frac{u}{z}\left(\frac{x^{\prime}}{\alpha_{0}}\right)^{2}\right]\right\} \text {. }
$$


Since in the definition of $\Psi_{\mathrm{WP}}(x, t)$, according to equation (14), only $G$ actually depends on $x$ and $t$, the kernel $G$, as defined in equation (16), must also fulfil the time-dependent SE. Inserting (16) into the SE (1) shows that $z(t)$ and $u(t)$ not only fulfil the same equation of motion as $\eta(t)$ and $\lambda(t)$ but, in addition, are also uniquely connected via the relation

$$
\dot{z} u-\dot{u} z=1 \text {. }
$$

Expressing $u$ and $z$ in polar coordinates according to $u=\alpha \cos \varphi$ and $z=\alpha \sin \varphi$ shows that this coupling is identical to relation (13) that connects the amplitude and phase of $\lambda$. From equation (17) it also follows that, e.g., with the knowledge of $z$, the quantity $u$ can be calculated as

$$
u=-z \int^{t} \frac{1}{z^{2}} d t^{\prime}
$$

The last necessary step is to explicitly perform the integration in (14), using (15) and (16), to yield the WP solution in the form

$$
\Psi_{\mathrm{WP}}(x, t)=\left(\frac{m}{\pi \hbar}\right)^{1 / 4}\left(\frac{1}{u+i z}\right)^{1 / 2} \exp \left\{\frac{i m}{2 \hbar}\left[\frac{\dot{z}}{z} x^{2}-\frac{\left(x-\frac{p_{0} \alpha_{0}}{m} z\right)^{2}}{z(u+i z)}\right]\right\} .
$$

Comparison with the same WP, written in the form given in equation (2), shows that the relations

$$
z=\frac{m}{\alpha_{0} p_{0}} \eta(t)
$$

and

$$
\frac{2 \hbar}{m} y=\frac{\dot{z}}{z}-\frac{1}{z \lambda}=\frac{\dot{\lambda}}{\lambda}
$$

are valid, where $\lambda=u+i z$ is identical to our linearization variable and equation (17) has been applied. So, from equation (18), it then follows that the imaginary part of $\lambda$ is, apart from a constant factor, just the particle trajectory.

The equivalence between deriving the time-dependent Green's function via a Gaussian ansatz or via Feynman's path integral method has been mentioned in [6], where also the relation to the Ermakov invariant is considered.

In conclusion, one can say that the complex quantity $\lambda$ contains the particle as well as the wave aspects of the system. In polar coordinates, the absolute value $\alpha$ of $\lambda$ is directly connected with the quantum mechanical position uncertainty; in cartesian coordinates, the imaginary part of $\lambda$ is directly proportional to the classical particle trajectory $\eta$. Absolute value and phase, or real and imaginary parts, of $\lambda$ are not independent of each other but uniquely connected via the conservation laws (13) and (17), respectively.

\subsection{Quantum-classical connection}

Further insight into the relation between the classical and quantum dynamics of the system can be gained by rewriting the invariant (10), with the help of equation (18), in terms of $z$ and $\dot{z}$ instead of $\eta$ and $\dot{\eta}$,

$$
I_{\mathrm{L}}=\frac{1}{2}\left(\frac{\alpha_{0} p_{0}}{m}\right)^{2}\left[(\dot{z} \alpha-z \dot{\alpha})^{2}+\left(\frac{z}{\alpha}\right)^{2}\right]=\text { const. }
$$


Since $\frac{z}{\alpha}=\sin \varphi$, for $I_{\mathrm{L}}$ to be constant it is necessary that $(\dot{z} \alpha-z \dot{\alpha})^{2}=\cos ^{2} \varphi=\left(\frac{u}{\alpha}\right)^{2}$ is valid. So, up to a \pm sign, one obtains

$$
u=\dot{z} \alpha^{2}-z \dot{\alpha} \alpha=\alpha^{2}\left(\dot{z}-\frac{\dot{\alpha}}{\alpha} z\right)
$$

With this form of $u$, a certain asymmetry in the exponent of the Feynman kernel (16), namely $\frac{\dot{z}}{z}$ as coefficient of $x^{2}$ compared to $\frac{1}{\alpha_{0}^{2}} \frac{u}{z}$ as coefficient of the initial quantity $x^{\prime 2}$, can be explained. According to (19), $\frac{\dot{z}}{z}$ can be written as

$$
\frac{\dot{z}}{z}=\frac{1}{\alpha^{2}(t)} \frac{u}{z}+\frac{\dot{\alpha}}{\alpha}
$$

This shows that, in the case when $\alpha$ is time-dependent, not only $\alpha_{0}^{2}$ must be replaced by $\alpha^{2}(t)$, but also an additional term $\frac{\dot{\alpha}}{\alpha}$ must be taken into account. For constant $\alpha=\alpha_{0}$ or at $t=0$, relation (20) reduces to

$$
\frac{\dot{z}}{z}=\frac{1}{\alpha_{0}^{2}} \frac{u}{z} \quad \text { or } \quad u=\alpha_{0}^{2} \dot{z}=\frac{m \alpha_{0}}{p_{0}} \dot{\eta}
$$

i.e., $u$ is simply proportional to $\dot{\eta}$. Note the explicit occurrence of $\alpha_{0}$, the initial position uncertainty, because it has important consequences for the time-dependence of $\alpha(t)$. Inserting $u$, as given in (21), into $\alpha^{2}(t)=u^{2}+z^{2}$ yields (with $v_{0}=\frac{p_{0}}{m}$ and $\beta_{0}=\frac{1}{\alpha_{0}^{2}}$ )

$$
\alpha^{2}(t)=\frac{\alpha_{0}^{2}}{v_{0}^{2}}\left[\dot{\eta}^{2}+\beta_{0}^{2} \eta^{2}\right]=\frac{2 m}{\hbar}\left\langle\tilde{x}^{2}\right\rangle
$$

This shows that the quantum mechanical uncertainty of position (at any time $t$ ) can be expressed solely in terms of the classical trajectory $\eta(t)$ and the corresponding velocity $\dot{\eta}(t)$, if the initial velocity $v_{0}$ and the initial position uncertainty, expressed by $\alpha_{0}$ are known.

This explains why Feynman's procedure [7] of deriving his kernel based only on the classical Lagrangian provides the correct time-evolution of the system since the time-dependence enters only via the classical variables $\eta(t)$ and $\dot{\eta}(t)$. However, the importance of the initial position uncertainty $\alpha_{0}$ for the quantum dynamics should not be underestimated.

The influence of the initial uncertainty becomes clear if one inserts the expressions for $\eta(t)$ and $\dot{\eta}(t)$ into equation (22).

a) For the free motion, one obtains with $\eta=v_{0} t, \dot{\eta}=v_{0}$ :

$$
\alpha_{\mathrm{fr}}^{2}=\alpha_{0}^{2}\left[1+\left(\beta_{0} t\right)^{2}\right] ;
$$

b) for the $\mathrm{HO}$ with $\eta=\frac{v_{0}}{\omega} \sin \omega t$ and $\dot{\eta}=v_{0} \cos \omega t$, equation (22) yields

$$
\alpha_{\mathrm{HO}}^{2}=\alpha_{0}^{2}\left\{\cos ^{2} \omega t+\left(\frac{\beta_{0}}{\omega} \sin \omega t\right)^{2}\right\} .
$$

Only if the initial state is the ground state, is $\beta_{0}=\frac{\hbar}{2 m\left\langle\tilde{x}^{2}\right\rangle_{0}}=\frac{1}{\alpha_{0}^{2}}=\omega$ valid and, hence, $\alpha=\alpha_{0}$, i.e., the WP width remains constant; in all other cases it oscillates. This oscillating WP width corresponds to the general solution of the Riccati equation (4) and yields in the limit $\omega \rightarrow 0$ the correct spreading WP width of the free motion WP,

$$
\lim _{\omega \rightarrow 0} \alpha_{\mathrm{HO}}^{2}(t)=\alpha_{0}^{2}\left[1+\left(\beta_{0} t\right)^{2}\right]=\alpha_{\mathrm{fr}}^{2}(t),
$$


whereas the WP usually presented, with constant width $\alpha_{0}=\left(\frac{1}{\omega}\right)^{1 / 2}$, provides in this limit only a plane wave, no WP!

The time-derivative of $\alpha^{2}$, or,

$$
\dot{\alpha} \alpha=\frac{\alpha_{0}^{2}}{v_{0}^{2}} \dot{\eta}\left[\beta_{0}^{2} \eta+\ddot{\eta}\right]=\frac{\alpha_{0}^{2}}{v_{0}^{2}} \dot{\eta}\left[\beta_{0}^{2} \eta-\frac{\partial}{\partial \eta} V(\eta)\right],
$$

respectively, only vanishes if the term in square brackets is equal to zero, which depends on $\frac{\partial}{\partial \eta} V(\eta)$. Therefore, for $V=0(\ddot{\eta}=0), \dot{\alpha} \neq 0$ is always valid; for the $\mathrm{HO}\left(\ddot{\eta}=-\omega^{2} \eta\right)$, $\dot{\alpha}=0$ is only valid for $\beta_{0}=\frac{1}{\alpha_{0}^{2}}=\omega$, otherwise, and in particular for $\omega=\omega(t)$ (which describes, e.g., the motion of an ion in a Paul trap [8]), $\dot{\alpha} \neq 0$ always holds. So, obviously the initial value $\alpha_{0}$ of the position uncertainty plays an important role in the qualitative behaviour of the quantum aspect of the dynamics. A more detailed discussion of this problem can be found in [9], also including dissipative effects.

\section{Ermakov invariant and Wigner function}

In the previous section, it has been shown how the real and imaginary parts (in cartesian coordinates) of the complex variable $\lambda(t)$, that allows the linearization of the complex Riccati equation (4), enter the Feynman kernel that describes the transformation of an initial quantum state into a state at a later time $t$ as time-dependent parameters. But, also in polar coordinates $\alpha$ and $\varphi$ (which are not independent of each other but related via $\left.\dot{\varphi}=\frac{1}{\alpha^{2}}\right), \lambda(t)$ is related, via the Ermakov invariant (10), with another description of quantum systems that shows close similarity with the classical phase space description of dynamical systems, namely the Wigner function. In order to show this connection the invariant $I_{\mathrm{L}}$ shall be written explicitly in the form

$$
I_{\mathrm{L}}=\frac{1}{2}\left[\left(\dot{\alpha}^{2}+\frac{1}{\alpha^{2}}\right) \eta^{2}-2 \alpha \dot{\alpha} \eta \dot{\eta}+\alpha^{2} \dot{\eta}^{2}\right]
$$

with terms bilinear in $\eta$ and $\dot{\eta}$. How the coefficients of these terms are related with the quantum uncertainties, and thus with $\alpha, \varphi$ and $\lambda$, follows from

$$
\begin{aligned}
& \lambda \lambda^{*}=\alpha^{2}=\frac{m}{2 \hbar y_{\mathrm{I}}}=\frac{2 m}{\hbar}\left\langle\tilde{x}^{2}\right\rangle_{\mathrm{L}}, \\
& \dot{\lambda} \dot{\lambda}^{*}=\dot{\alpha}^{2}+\alpha^{2} \dot{\varphi}^{2}=\frac{\hbar}{m} \frac{y_{\mathrm{R}}^{2}+y_{\mathrm{I}}^{2}}{y_{\mathrm{I}}}=\frac{\hbar m}{2}\left\langle\tilde{p}^{2}\right\rangle_{\mathrm{L}}, \\
& \frac{\partial}{\partial t}\left(\lambda \lambda^{*}\right)=2 \dot{\alpha} \alpha=2\left(\frac{y_{\mathrm{R}}}{y_{\mathrm{I}}}\right)=\frac{2}{\hbar}\left\langle[\tilde{x}, \tilde{p}]_{+}\right\rangle_{\mathrm{L}}=\frac{2}{\hbar}\langle\tilde{x} \tilde{p}+\tilde{p} \tilde{x}\rangle_{\mathrm{L}} .
\end{aligned}
$$

With the help of these relations, the invariant takes the form

$$
\begin{aligned}
I_{\mathrm{L}} & =\frac{1}{2}\left[\dot{\lambda} \dot{\lambda}^{*} \eta^{2}-\frac{\partial}{\partial t}\left(\lambda \lambda^{*}\right) \eta \dot{\eta}+\lambda \lambda^{*} \dot{\eta}^{2}\right] \\
& =\frac{1}{m \hbar}\left[\left\langle\tilde{p}^{2}\right\rangle_{\mathrm{L}} \eta^{2}-\left\langle[\tilde{x}, \tilde{p}]_{+}\right\rangle_{\mathrm{L}} \eta(m \dot{\eta})+\left\langle\tilde{x}^{2}\right\rangle_{\mathrm{L}}(m \dot{\eta})^{2}\right] .
\end{aligned}
$$

The connection with the Wigner function becomes obvious if one performs the Wigner transformation [10] of our WP (2) according to

$$
W(x, p, t)=\frac{1}{2 \pi \hbar} \int_{-\infty}^{+\infty} d q e^{i p q / \hbar} \Psi_{\mathrm{WP}}^{*}\left(x+\frac{q}{2}, t\right) \Psi_{\mathrm{WP}}\left(x-\frac{q}{2}, t\right)
$$


yielding the Wigner function in the form

$$
W(x, p, t)=\frac{1}{\pi \hbar} \exp \left\{-2\left(\frac{y_{\mathrm{I}}^{2}+y_{\mathrm{R}}^{2}}{y_{\mathrm{I}}}\right) \tilde{x}^{2}-\frac{\tilde{p}^{2}}{2 \hbar^{2} y_{\mathrm{I}}}+\frac{2}{\hbar}\left(\frac{y_{\mathrm{R}}}{y_{\mathrm{I}}}\right) \tilde{x} \tilde{p}\right\} .
$$

Using relations $(23)-(25)$, this can be expressed as

$$
W(x, p, t)=\frac{1}{\pi \hbar} \exp \left\{-\frac{2}{\hbar^{2}}\left[\left\langle\tilde{p}^{2}\right\rangle_{\mathrm{L}} \tilde{x}^{2}-\left\langle[\tilde{x}, \tilde{p}]_{+}\right\rangle_{\mathrm{L}} \tilde{x} \tilde{p}+\left\langle\tilde{x}^{2}\right\rangle_{\mathrm{L}} \tilde{p}^{2}\right]\right\} .
$$

In particular, at the origin of the phase space, i.e., for $x=0$ and $p=0$, where $\tilde{x}^{2} \rightarrow \eta^{2}$, $\tilde{p}^{2} \rightarrow(m \dot{\eta})^{2}$ and $\tilde{x} \tilde{p} \rightarrow m \eta \dot{\eta}$, one obtains

$$
W(0,0, t)=\frac{1}{\pi \hbar} \exp \left\{-\frac{2 m}{\hbar} I_{\mathrm{L}}\right\}=\text { const }
$$

with $I_{\mathrm{L}}$ as given in (26) which fulfils $\frac{\partial}{\partial t} W=0$ since $I_{\mathrm{L}}$ is an invariant. For $x \neq 0$ and $p \neq 0$, $W(x, p, t)$ takes the form (27) and the equation of motion is given, as expected, by

$$
\frac{\partial}{\partial t} W(x, p, t)=-\frac{p}{m} \frac{\partial W}{\partial x}+\frac{\partial V}{\partial x} \frac{\partial W}{\partial p},
$$

i.e., a continuity equation for an incompressible medium, also called Liouville equation in phase space.

Further details, also concerning the different forms to express the Ermakov invariant in the exponent of the Wigner function and the physical interpretation of these forms is given in [11]. Other attempts to construct connections between the Ermakov invariant and the Wigner function can be found in [6].

\section{Riccati and Ermakov equations in time-independent quantum mechanics}

\subsection{Factorization of the invariant and creation/annihilation operators}

Formal similarities between the Ermakov invariant and the algebraic treatment of the HO using creation and annihilation operators (or complex normal modes, in the classical case) and its generalization in the formalism of SUSY can be found if $I_{\mathrm{L}}$ is written in a form that allows for factorization

$$
I_{\mathrm{L}}=\frac{1}{2} \alpha^{2}\left[\left(\dot{\eta}-\frac{\dot{\alpha}}{\alpha} \eta\right)^{2}+\left(\frac{\eta}{\alpha^{2}}\right)^{2}\right]=\frac{1}{2} \alpha^{2} A A^{*}
$$

with

$$
A=\left(\dot{\eta}-\frac{\dot{\alpha}}{\alpha} \eta\right)-i \frac{1}{\alpha^{2}} \eta=\dot{\eta}-\left(\frac{2 \hbar}{m} y\right) \eta
$$

and

$$
A^{*}=\left(\dot{\eta}-\frac{\dot{\alpha}}{\alpha} \eta\right)+i \frac{1}{\alpha^{2}} \eta=\dot{\eta}-\left(\frac{2 \hbar}{m} y^{*}\right) \eta .
$$

For the HO with time-independent frequency $\omega_{0}$ and constant WP width $\alpha_{0}$, the real part of $\left(\frac{2 \hbar}{m} y\right)$ vanishes $(\dot{\alpha}=0)$ and the imaginary part is simply $\frac{1}{\alpha_{0}^{2}}=\dot{\varphi}=\omega_{0}$, so equations (28) and (29) turn into

$$
A_{0}=\dot{\eta}-i \omega_{0} \eta, \quad A_{0}^{*}=\dot{\eta}+i \omega_{0} \eta
$$


These expressions are, up to constant factors, identical with the complex normal modes or, in the quantized form, with the creation and annihilation operators of the HO. In this context, it should be mentioned that the Ermakov invariant has also been quantized, where $\alpha$ and $\dot{\alpha}$ remain c-numbers, whereas position $\eta$ and momentum $m \dot{\eta}$ are, following the rules of canonical quantization, replaced by the corresponding operators, i.e., $\eta \rightarrow q_{\mathrm{op}}=q, m \dot{\eta} \rightarrow p_{\mathrm{op}}=\frac{\hbar}{i} \frac{\partial}{\partial q}$ (for details see, e.g., [12]). Expressions (30) would then turn into the operators

$$
A_{0, \mathrm{op}}=\frac{1}{m} p_{\mathrm{op}}-i \omega_{0} q, \quad A_{0, \mathrm{op}}^{*}=\frac{1}{m} p_{\mathrm{op}}+i \omega_{0} q .
$$

For comparison, the Hamiltonian operator of the $\mathrm{HO}$ can be written as

$$
H_{\mathrm{op}, \mathrm{HO}}=\frac{1}{2 m} p_{\mathrm{op}}^{2}+\frac{m}{2} \omega_{0}^{2} q^{2}=\hbar \omega_{0}\left(\hat{b}^{+} \hat{b}^{-}+\frac{1}{2}\right)
$$

with the creation/annihilation operators

$$
\hat{b}^{ \pm}=\mp i \sqrt{\frac{m}{2 \hbar \omega_{0}}}\left(\frac{p_{\mathrm{op}}}{m} \pm i \omega_{0} q\right)=\sqrt{\frac{m}{2 \hbar \omega_{0}}}\left(\omega_{0} q \mp i \frac{p_{\mathrm{op}}}{m}\right) .
$$

Comparison shows that

$$
\alpha_{0} A_{0, \mathrm{op}}=-i \sqrt{\frac{2 \hbar}{m}} \hat{b}^{-}, \quad \alpha_{0} A_{0, \mathrm{op}}^{*}=+i \sqrt{\frac{2 \hbar}{m}} \hat{b}^{+} .
$$

So, $A$ and $A^{*}$ (or $A_{\mathrm{op}}$ and $A_{\mathrm{op}}^{*}$ ) are generalizations where the constant factor $\pm i \omega_{0}$, in front of $q$, is replaced by the complex time-dependent functions $\left(\frac{2 \hbar}{m} y\right)$ or $\left(\frac{2 \hbar}{m} y^{*}\right)$, respectively. The factorization of the dynamical invariant and the relation to creation/annihilation operators is also discussed in [6].

\subsection{Riccati and SUSY}

A different generalization of the creation/annihilation operators is found in SUSY where, essentially, the term linear in the coordinate $q$ is replaced by a function of $q$, the so-called "superpotential" $W(q)$, leading to the operators

$$
B^{ \pm}=\frac{1}{\sqrt{2}}\left[W(q) \mp i \frac{p_{\mathrm{op}}}{\sqrt{m}}\right] \text {. }
$$

In this case, the term $\omega_{0} q$ with constant $\omega_{0}$ is replaced by a real, position-dependent function $W(q)$. The operators $B^{ \pm}$fulfil the commutator and anti-commutator relations

$$
\left[B^{-}, B^{+}\right]_{-}=\frac{\hbar}{\sqrt{m}} \frac{d W}{d q}, \quad\left\{B^{-}, B^{+}\right\}_{+}=W^{2}+\frac{p_{\mathrm{op}}^{2}}{m} .
$$

The supersymmetric Hamiltonian

$$
H_{\mathrm{SUSY}}=\left(\begin{array}{cc}
H_{1} & 0 \\
0 & H_{2}
\end{array}\right)
$$

can be expressed with the help of $B^{ \pm}$in the form

$$
H_{1}=B^{+} B^{-}=-\frac{\hbar^{2}}{2 m} \frac{d^{2}}{d q^{2}}+V_{1}(q), \quad H_{2}=B^{-} B^{+}=-\frac{\hbar^{2}}{2 m} \frac{d^{2}}{d q^{2}}+V_{2}(q) .
$$


A detailed discussion of the formalism of SUSY can be found, e.g., in [13]; for our discussion, only the aspects mentioned in the following will be necessary. Important in this context is that the supersymmetric partner potentials $V_{1}(q)$ and $V_{2}(q)$ fulfil real Riccati equations, which follows directly from the definition of $B^{ \pm}$, i.e.,

$$
\begin{aligned}
& V_{1}=\frac{1}{2}\left[W^{2}-\frac{\hbar}{\sqrt{m}} \frac{d W}{d q}\right] \\
& V_{2}=\frac{1}{2}\left[W^{2}+\frac{\hbar}{\sqrt{m}} \frac{d W}{d q}\right] .
\end{aligned}
$$

The energy spectra of $H_{1}$ and $H_{2}$ are identical apart from the ground state. $H_{1}$ has the ground state $E_{0}^{(1)}=0$, whereas the ground state $E_{0}^{(2)}$ of $H_{2}$ is identical with the first excited state $E_{1}^{(1)}$ of $H_{1}$. The ground state wave function of $H_{1}, \Psi_{0}^{(1)}$, has no node and determines the superpotential via

$$
W=-\frac{\hbar}{\sqrt{m}} \frac{\frac{d}{d q} \Psi_{0}^{(1)}}{\Psi_{0}^{(1)}} .
$$

From equations (32) and (33), then, the partner potentials $V_{1}$ and $V_{2}$ follow. On the other hand, $\Psi_{0}^{(1)}$ is connected with $V_{1}$ via the solution of the equation $H_{1} \Psi_{0}^{(1)}=0$, i.e.,

$$
H_{1} \Psi_{0}^{(1)}=-\frac{\hbar^{2}}{2 m} \frac{d^{2}}{d q^{2}} \Psi_{0}^{(1)}+\frac{1}{2}\left[W^{2}-\frac{\hbar}{\sqrt{m}} \frac{d W}{d q}\right] \Psi_{0}^{(1)}=E_{0}^{(1)} \Psi_{0}^{(1)}=0 .
$$

The connection between the spectra of $H_{1}$ and $H_{2}$, i.e. $E_{n}^{(1)}$ and $E_{n}^{(2)}$, and the corresponding wave functions, $\Psi_{n}^{(1)}$ and $\Psi_{n}^{(2)}$, is determined via the generalized creation/annihilation operators $B^{ \pm}$according to

$$
\Psi_{n+1}^{(1)}=\frac{1}{\sqrt{E_{n}^{(2)}}} B^{+} \Psi_{n}^{(2)} \quad \text { and } \quad \Psi_{n}^{(2)}=\frac{1}{\sqrt{E_{n+1}^{(1)}}} B^{-} \Psi_{n+1}^{(1)},
$$

where $B^{+}$creates a node and $B^{-}$annihilates a node in the wave function. So, e.g., the first excited state $\Psi_{1}^{(1)}$ of $H_{1}$ (which has one node) can be obtained from the ground state $\Psi_{0}^{(2)}$ of $H_{2}$ (which has no node) by applying $B^{+}$onto it as described in (34).

In order to obtain the higher eigenvalues and eigenfunctions, $\Psi_{0}^{(1)}$ in the definition of $W \equiv W_{1}$ must be replaced by $\Psi_{0}^{(2)}$, leading to $W_{2}=-\frac{\hbar}{\sqrt{m}} \frac{\frac{d}{d q} \Psi_{0}^{(2)}}{\Psi_{0}^{(2)}}$ etc., i.e.,

$$
W_{s}=-\frac{\hbar}{\sqrt{m}} \frac{\frac{d}{d q} \Psi_{0}^{(s)}}{\Psi_{0}^{(s)}}
$$

with the corresponding operators

$$
B_{s}^{ \pm}=\frac{1}{\sqrt{2}}\left[W_{s} \mp \frac{\hbar}{\sqrt{m}} \frac{d}{d q}\right]
$$

thus creating a hierarchy that provides all eigenvalues and eigenfunctions of the Hamiltonians $H_{1}$ and $\mathrm{H}_{2}$.

In the context of this paper, only two systems with exact analytic solutions shall be considered explicitly, namely, the one-dimensional HO (with constant frequency $\omega=\omega_{0}$ ) and the Coulomb problem. The latter case, a three-dimensional system with spherical symmetry $(V(\vec{r})=V(r)=$ 
$-\frac{e^{2}}{r}$ ), can be reduced to an essentially one-dimensional problem via separation of radial and angular parts. Using the ansatz $\Phi_{n l m}(\vec{r})=\frac{1}{r} \Psi_{n l}(r) Y_{l m}(\vartheta, \varphi)=R(r) Y_{l m}(\vartheta, \varphi)$ for the wave function (with $n=$ total quantum number, $l=$ azimuthal quantum number, $m=$ magnetic quantum number, $r, \vartheta, \varphi=$ polar coordinates), the energy eigenvalues $E_{n}$ of the system can be obtained from the radial SE

$$
\left\{-\frac{\hbar^{2}}{2 m} \frac{d^{2}}{d r^{2}}+V_{\text {eff }}\right\} \Psi_{n l}(r)=E_{n} \Psi_{n l}(r)
$$

with the effective potential

$$
V_{\mathrm{eff}}=V(r)+\frac{l(l+1) \hbar^{2}}{2 m r^{2}}=-\frac{e^{2}}{r}+\frac{l(l+1) \hbar^{2}}{2 m r^{2}} .
$$

The superpotential $W$, the energy eigenvalues $E_{n}$ and the supersymmetric potential $V_{1}$ for the systems under consideration are given by:

a) HO: $V(q)=\frac{m}{2} \omega^{2} q^{2}$ (eigenfunctions $\Psi_{n}(q)$ : Hermite polynomials)

$$
\begin{aligned}
& W=\omega q, \\
& E_{n}=\hbar \omega\left(n+\frac{1}{2}\right), \\
& V_{1}=\frac{m}{2} \omega^{2} q^{2}-\frac{\hbar}{2} \omega=V(q)-E_{0} .
\end{aligned}
$$

b) Coulomb potential: $V(r)=-\frac{e^{2}}{r}$ (eigenfunctions $\Psi_{n l}(r)$ : Laguerre polynomials)

$$
\begin{aligned}
& W=\frac{\sqrt{m} e^{2}}{(l+1) \hbar}-\frac{(l+1) \hbar}{\sqrt{m} r} \\
& E_{n^{\prime}}=-\frac{m c^{2}}{2}\left(\frac{e^{2}}{\hbar c}\right)^{2} \frac{1}{\left(n^{\prime}+l+1\right)^{2}}, \\
& V_{1}=-\frac{e^{2}}{r}+\frac{l(l+1) \hbar^{2}}{2 m r^{2}}+\frac{m c^{2}}{2}\left(\frac{e^{2}}{\hbar c}\right)^{2} \frac{1}{(l+1)^{2}}=V_{\text {eff }}-E_{0} .
\end{aligned}
$$

In the second case, the radial quantum number $n^{\prime}$ occurs which indicates the number of nodes in the wave function and is connected with the total quantum number $n$, that actually characterizes the energy eigenvalue, via $n=n^{\prime}+l+1$.

Particularly the quantities $V_{1}$, given in equations (36) and (37), shall be compared with similar expressions obtained in the next subsection where a nonlinear formulation of time-independent quantum mechanics is presented.

\subsection{Ermakov and nonlinear time-independent quantum mechanics}

In the following discussion of a nonlinear formulation of quantum mechanics, that is essentially based on the work of Reinisch [2], formal similarities with SUSY (in the time-independent case) and the complex Riccati formalism (in the time-dependent case) shall be pointed out.

Starting point is Madelung's hydrodynamic formulation of quantum mechanics [14] that uses the polar ansatz

$$
\Psi(\vec{r}, t)=a(\vec{r}) \exp \left\{-\frac{i}{\hbar} S(\vec{r}, t)\right\}
$$


for the wave function $\Psi(\vec{r}, t)$, turning the linear SE (1) into two coupled equations for the amplitude $a(\vec{r})$ and the phase $S(\vec{r}, t)$, i.e., the Hamilton-Jacobi-type equation

$$
\frac{\partial}{\partial t} S+\frac{1}{2 m}(\nabla S)^{2}+V-\frac{\hbar^{2}}{2 m} \frac{\Delta a}{a}=0
$$

(with $\nabla=$ Nabla operator, $\Delta=$ Laplace operator) and the continuity equation

$$
\frac{\partial}{\partial t} a^{2}+\frac{1}{m} \nabla\left(a^{2} \nabla S\right)=0
$$

where $a^{2}=\Psi^{*} \Psi=\varrho(\vec{r}, t)$ is the usual probability density. For stationary states, the energy of the system is related with the action $S$ via $\frac{\partial}{\partial t} S=-E=$ const and the density is time-independent, i.e.,

$$
\frac{\partial}{\partial t} a^{2}=0
$$

where it subsequently follows that the second term on the lhs of equation (39) must also vanish. In the usual textbook treatment, this is achieved by taking $\nabla S=0$, thus turning equation (38) into the conventional time-independent linear SE

$$
-\frac{\hbar^{2}}{2 m} \Delta a+V a=E a .
$$

This is, however, not the only possibility of fulfilling equation (39) since, also for $\nabla S \neq 0$, this can be accomplished if only

$$
\nabla S=\frac{C}{a^{2}}
$$

with constant $C$. In this case, equation (38) takes the form of a nonlinear Ermakov equation

$$
\Delta a+\frac{2 m}{\hbar}(E-V) a=\left(\frac{C}{\hbar}\right)^{2} \frac{1}{a^{3}} .
$$

The corresponding complex Riccati equation, equivalent to equation (4) in the time-dependent case, is given here by

$$
\nabla\left(\frac{\nabla \Psi}{\Psi}\right)+\left(\frac{\nabla \Psi}{\Psi}\right)^{2}+\frac{2 m}{\hbar}(E-V)=0,
$$

where the following substitutions must be made

$$
\frac{\partial}{\partial t} \leftrightarrow \nabla, \quad\left(\frac{2 \hbar}{m} y\right)=\frac{\dot{\lambda}}{\lambda} \leftrightarrow \frac{\nabla \Psi}{\Psi}, \quad \lambda=\alpha e^{i \varphi} \leftrightarrow \Psi=a e^{i \frac{S}{\hbar}}
$$

Considering first the one-dimensional $\mathrm{HO}$, and introducing the dimensionless variable $\zeta$ via $\zeta=\left|k_{0}\right| q$ with $\hbar k_{0}=p_{0}= \pm \sqrt{2 m E}, \tilde{V}(\zeta)=V[q(\zeta)]$ and $\ddot{a}=\frac{d^{2}}{d \zeta^{2}} a$, equation (42) acquires the familiar form

$$
\ddot{a}+\left(1-\frac{\tilde{V}}{E}\right) a=\frac{1}{a^{3}} .
$$

A similar formulation of the time-independent SE in terms of this equation, but within a different context and different applications has also been given in [15]. In another paper [16] the relation between the Ermakov equation (44) and the time-independent SE has been extended 
to also include magnetic field effects. The nonlinear differential equation (44) has also been used to obtain numerical solutions of the time-independent SE for single and double-minimum potentials as well as for complex energy resonance states; for details see [17]. Here we want to concentrate on the similarities between the time-dependent and time-independent situation, in particular with respect to SUSY, as mentioned in Sections 5.1 and 5.2.

Following the method described in [2], from the real solution $a_{\mathrm{NL}}(\zeta)$ of this nonlinear Ermakov equation (44) the complex solution $a_{\mathrm{L}}(\zeta)$ of the linear $\mathrm{SE}(40)$ can be obtained via

$$
a_{\mathrm{L}}(\zeta)=a_{\mathrm{NL}}(\zeta) \exp \left\{-\frac{i}{\hbar} S\right\}=a_{\mathrm{NL}}(\zeta) \exp \left\{-i \int_{\zeta_{0}}^{\zeta} d \zeta^{\prime} \frac{1}{a_{\mathrm{NL}}^{2}\left(\zeta^{\prime}\right)}\right\}
$$

from which a real (not normalized) solution of the time-independent SE can be constructed according to

$$
\tilde{a}_{\mathrm{L}}(\zeta)=\Re\left[a_{\mathrm{L}}(\zeta)\right]=\frac{1}{2}\left[a_{\mathrm{NL}} e^{\frac{i}{\hbar} S}+a_{\mathrm{NL}} e^{-\frac{i}{\hbar} S}\right]=a_{\mathrm{NL}} \cos \left(\int_{\zeta_{0}}^{\zeta} d \zeta^{\prime} \frac{1}{a_{\mathrm{NL}}^{2}\left(\zeta^{\prime}\right)}\right) .
$$

So far, the energy $E$ occurring in equation (44) is still a free parameter that can take any value. However, solving equation (44) numerically for arbitrary values of $E$ leads, in general, to solutions $a_{\mathrm{NL}}$ that diverge for increasing $\zeta$. Only if the energy $E$ is appropriately tuned to any eigenvalue $E_{n}$ of equation (40) does this divergence disappear and the integral in the cosine of equation (46) takes for $\zeta \rightarrow \infty$ exactly the value $\frac{\pi}{2}$, i.e., the cosine vanishes at infinity. So, the quantization condition that is usually obtained from the requirement of the truncation of an infinite series in order to avoid divergence of the wave function is, in this case, obtained from the requirement of nondiverging solutions of the nonlinear Ermakov equation (44) by variation of the parameter $E$. This has been numerically verified in the case of the one-dimensional HO and the Coulomb problem and there is the conjecture that this property is "universal" in the sense that it does not depend on the potential $V$ (see [2]).

For comparison with the situation in SUSY, the HO and the Coulomb problem can be written in the form:

a) HO: with $\mu=\left(\frac{\hbar \omega}{2 E}\right)^{2}, E=E_{n}=\left(n+\frac{1}{2}\right) \hbar \omega \rightarrow \mu_{n}=\frac{1}{(2 n+1)^{2}}$ and $\mu \zeta^{2}=\frac{\frac{m}{2} \omega^{2} q^{2}}{E}=\frac{V}{E}$ follows:

$$
\ddot{a}+\left(1-\mu \zeta^{2}\right) a=\ddot{a}+\left(1-\frac{\tilde{V}}{E}\right) a=\ddot{a}-\frac{U_{n}}{E} a=\frac{1}{a^{3}},
$$

where

$$
U_{n}=\frac{m}{2} \omega^{2} q^{2}-\hbar \omega\left(n+\frac{1}{2}\right)=V(q)-E_{n} .
$$

b) Coulomb problem: with $a(r, \vartheta, \varphi)=R(r) Y_{l m}(\vartheta, \varphi)$ the radial part can be separated and, with the dimensionless variable $\zeta=\left|k_{0}\right| r$ with now $\hbar k_{0}=p_{0}= \pm \sqrt{2 m(-E)}(E<0)$, the radial wave function can be written as $X(\zeta)=r(\zeta) X[r(\zeta)]$, which corresponds to $\Psi_{n l}(r)$ in SUSY. This function obeys, again, an Ermakov equation, namely

$$
\ddot{X}+\left(\frac{\tilde{W}}{E}-1\right) X=\ddot{X}+\frac{U_{n^{\prime}}}{E} X=\frac{1}{X^{3}},
$$

where

$$
\tilde{W}(\zeta)=\tilde{V}[r(\zeta)]+\frac{l(l+1) \hbar^{2}}{2 m r^{2}(\zeta)} \hat{=} V_{\text {eff }}
$$


is just the effective potential $V_{\text {eff }}$ from SUSY (see equations (35) and (37)) and $E=E_{n}=-\frac{m e^{4}}{2 \hbar^{2} n^{2}}$ with $n=n^{\prime}+l+1$. The coefficient of the term linear in $X$ can again be expressed with the help of the potential-like expression $U_{n^{\prime}}$ as

$$
U_{n^{\prime}}=-\frac{e^{2}}{r}+\frac{l(l+1) \hbar^{2}}{2 m r^{2}}+\frac{m c^{2}}{2}\left(\frac{e^{2}}{\hbar c}\right)^{2} \frac{1}{\left(n^{\prime}+l+1\right)^{2}}=V_{\text {eff }}-E_{n^{\prime}} .
$$

In both cases, the ground state $(n=0)$ wave functions are real, nodeless $\left(n^{\prime}=0\right)$ and the phase does not depend on spatial variables (i.e., $\nabla S=0$ ). Therefore, the rhs of equations (47) and (48) vanishes since $\frac{1}{a^{3}} \propto(\nabla S)^{2} a$ (similar for the Coulomb problem), i.e., the nonlinear Ermakov equations turn into the usual time-independent SEs. In this case, comparison shows that for the $\mathrm{HO}$ and the Coulomb problem, the potential-like terms $U_{0}$ are identical with the corresponding $V_{1}$ of SUSY. For $n>0$ and $n^{\prime}>0$, however, $U_{n}$ and $U_{n^{\prime}}$ are different from $V_{1}$ and describe higher excited states. In SUSY, these states can only be obtained from the hierarchy described in the previous subsection. Here, the price that must be paid to include these excited states is the nonlinearity on the rhs of equations (47) and (48).

Comparing the situation in this nonlinear formulation of time-independent quantum mechanics with SUSY and the time-dependent systems discussed in the first part of this paper, one can see the following similarities:

i) Comparison with SUSY:

The real superpotential $W=-\frac{\hbar}{\sqrt{m}}\left(\frac{\nabla \Psi_{0}}{\Psi_{0}}\right)$ is replaced by the complex "superpotential" $\mathcal{C}(q)=$ $-\frac{\hbar}{\sqrt{m}}\left[\left(\frac{\nabla|\Psi|}{|\Psi|}\right)+i \frac{\nabla S}{\hbar}\right]$, i.e., the ground state $\Psi_{0}$ is replaced by the absolute value $|\Psi|$ of any eigenstate and an additional imaginary part depending on the phase $\frac{S}{\hbar}$ of the wave function occurs, being responsible for the non-vanishing rhs of the Ermakov equations (47) and (48).

ii) Comparison with time-dependent $S E$ :

As mentioned before, the complex quantity $\mathcal{C}(q)$ can be compared with the time-dependent quantity $\left(\frac{2 \hbar}{m} y\right)$ fulfilling the complex Riccati equation $(4), \mathcal{C}(q)=-\frac{\hbar}{\sqrt{m}} \frac{\nabla \Psi}{\Psi} \leftrightarrow\left(\frac{2 \hbar}{m} y(t)\right)=\frac{\dot{\lambda}}{\lambda}$, or, in terms of real and imaginary parts, $\frac{\nabla|\Psi|}{|\Psi|} \leftrightarrow \frac{\dot{\alpha}}{\alpha}$ and $\frac{\nabla S}{\hbar} \propto \frac{1}{|\Psi|^{2}} \leftrightarrow \dot{\varphi} \propto \frac{1}{\alpha^{2}}$.

\section{Conclusions and perspectives}

As a result of this investigation, one can state that the Ermakov invariant is a quantity of central importance that connects different forms for the description of the dynamics of quantum systems, such as the time-dependent SE, the time-dependent Green's function (or Feynman kernel) and the time-dependent Wigner function. Unlike the classical Hamiltonian or Lagrangian, this invariant not only depends on the classical variables such as position and momentum, but also on the quantum uncertainties of these quantities contained in $\alpha$ and $\dot{\alpha}$. Therefore, the initial values of these quantities also play an important role for the time-evolution of the quantum system, as has been demonstrated in the discussion of the time-dependence of the WP width or position uncertainty. So, the time-evolution of a typical quantum mechanical property can be totally described if one only knows the classical trajectory $\eta$ and the classical velocity $\dot{\eta}$ (including their initial conditions) plus the initial position uncertainty. This traces quantum dynamics entirely back to the classical one plus the existence of an uncertainty principle.

So far, the discussion of time-dependent systems included only systems where the potential is at most quadratic in its variables (a similar treatment of the motion in a magnetic field is also possible; see, e.g., [18]). This might not be as restrictive as it seems at first sight since one may sometimes perform canonical transformations to reduce a given Hamiltonian to a quadratic form [19] which has been shown explicitly by Sarlet for some polynomial Hamiltonians. To what extent this method can also be applied in our case requires more detailed studies. Another way 
to extend considerably the class of Hamiltonians for which an exact invariant can be found is by making use of generalized canonical transformations [20]. Further generalizations of applying the Ermakov-type invariants also in the context of field-atom interactions, systems of coupled oscillators including damping and/or time-dependent masses and attempts of obtaining the corresponding Wigner functions can be found in [21]. For a further survey of two-dimensional problems in this context, see also [22].

Similar to the factorization of the Hamiltonian of the HO in terms of (classical) complex normal modes, or, (quantum mechanical) in terms of creation and annihilation operators $b^{ \pm}$, a factorization of the Ermakov invariant is possible that looks like a complex time-dependent generalization of this formalism. The major difference compared with the conventional case is the replacement of $\pm i \omega_{0}$ in front of the spatial variable $q$ in equation (31) by the complex timedependent quantity $\left(\frac{2 \hbar}{m} y\right)$, which fulfils the Riccati equation (4), or its conjugate complex $\left(\frac{2 \hbar}{m} y^{*}\right)$, respectively.

Another generalization of the creation/annihilation operator formalism of the HO, concerning the space-dependence instead of the time-dependence, is found in SUSY where the term linear in the spatial coordinate $q$ is replaced by a function of $q$, the so-called "superpotential" $W(q)$. The generalized creation and annihilation operators $B^{ \pm}$are obtained by replacing the term $\omega_{0} q$ in $b^{ \pm}$by (up to constants) $W(q)$, where $W(q)$ fulfils the real Riccati equations (32) and (33).

In a nonlinear formulation of time-independent quantum mechanics proposed by Reinisch [2], the amplitude of the wave function fulfils a real nonlinear (space-dependent) Ermakov equation, that can be connected with the time-independent SE written as a complex space-dependent Riccati equation (see (43)) together with a kind of conservation law (see (41)), similar to the conservation of "angular momentum" in the complex plane (see (13)) in the time-dependent case. In SUSY, the superpotential is initially determined by the amplitude of the ground state wave function (i.e., without any contribution from the phase) and the excited states can be obtained by some hierarchy based thereupon, in the nonlinear formulation of time-independent quantum mechanics, the ground state wave function is replaced by the absolute value of any eigenstate plus an imaginary part depending on the gradient of the phase of this state. Similar to the time-dependent situation, this looks like a complex generalization where, now, the real superpotential $W(q)$ is replaced by a complex term that not only depends on the amplitude of the wave function, but, due to an additional imaginary contribution, also on (the gradient of) its phase. Further clarification of these facts will be subject of forthcoming studies.

\section{Acknowledgements}

The author wishes to thank G. Reinisch for numerous encouraging and inspiring discussions.

\section{References}

[1] Schrödinger E., Quantisierung als Eigenwertproblem, Ann. Phys. 79 (1926), 361-376.

[2] Reinisch G., Nonlinear quantum mechanics, Phys. A 206 (1994), 229-252.

Reinisch G., Classical position probability distribution in stationary and separable quantum systems, Phys. Rev. A 56 (1997), 3409-3416.

[3] Ermakov V.P., Second-order differential equations, Conditions of complete integrability, Univ. Izv. Kiev 20 (1880), no. 9, 1-25.

[4] Milne W.E., The numerical determination of characteristic numbers, Phys. Rev. 35 (1930), 863-867. Pinney E., The nonlinear differential equation $y^{\prime \prime}+p(x) y+c y^{-3}=0$, Proc. Amer. Math. Soc. 1 (1950), 681. Lewis H.R., Classical and quantum systems with time-dependent harmonic-oscillator-type Hamiltonians, Phys. Rev. Lett. 18 (1967), 510-512. 
[5] Lutzky M., Noether's theorem and the time-dependent harmonic oscillator, Phys. Lett. A 68 (1979), 3-4. Ray J.R., Reid J.L., More exact invariants for the time-dependent harmonic oscillator, Phys. Lett. A 71 (1979), 317-318.

[6] Malkin I.A., Man'ko V.I., Trifonov D.A., Linear adiabatic invariants and coherent states, J. Math. Phys. 14 (1973), 576-582.

Markov M.A. (Editor), Invariants and evolution of nonstationary quantum systems, Proceedings of the Lebedev Physical Institute, Vol. 183, Nova Science, New York, 1989.

[7] Feynman R.P., Hibbs A.R., Quantum mechanics and path integrals, McGraw-Hill, New York, 1965.

[8] Schleich W.P., Quantum optics in phase space, Wiley-VCh, Berlin, Chapter 17, 2001.

[9] Schuch D., Moshinsky M., Connection between quantum-mechanical and classical time evolution via a dynamical invariant, Phys. Rev. A 73 (2006), 062111, 10 pages.

Schuch D., Connection between quantum-mechanical and classical time evolution of certain dissipative systems via a dynamical invariant, J. Math. Phys. 48 (2007), 122701, 19 pages.

[10] Wigner E.P., On the quantum correction for thermodynamical equilibrium, Phys. Rev. 40 (1932), 749-759. Hillery M. O’Connell R.F., Scully M.O., Wigner E.P., Distribution functions in physics: fundamentals, Phys. Rep. 106 (1984), 121-167.

[11] Schuch D., On the relation between the Wigner function and an exact dynamical invariant, Phys. Lett. A 338 (2005), 225-231.

[12] Lewis H.R., Riesenfeld W.B., An exact quantum theory of the time-dependent harmonic oscillator and of a charged particle in a time-dependent electromagnetic field, J. Math. Phys. 10 (1969), 1458-1473.

Hartley J.G., Ray J.R., Ermakov systems and quantum-mechanical superposition law, Phys. Rev. A 24 (1981), 2873-2876.

[13] Cooper F., Khare A., Sukhatme U., Supersymmetry in Quantum Mechanics, World Scientific, Singapore, 2001.

Kalka H., Soff G., Supersymmetrie, Teubner, Stuttgart, 1997.

[14] Madelung E., Quantentheorie in hydrodynamischer Form, Z. Phys. 40 (1926), 322-326.

[15] Lee R.A., Quantum ray equations, J. Phys. A: Math. Gen. 15 (1982), 2761-2774.

[16] Kaushal R.S., Quantum analogue of Ermakov systems and the phase of the quantum wave function, Intern. J. Theoret. Phys. 40 (2001), 835-847.

[17] Korsch H.J., Laurent H., Milne's differential equation and numerical solutions of the Schrödinger equation. I. Bound-state energies for single- and double minimum potentials, J. Phys. B: At. Mol. Phys. 14 (1981), $4213-4230$.

Korsch H.J., Laurent H. and Mohlenkamp, Milne's differential equation and numerical solutions of the Schrödinger equation. II. Complex energy resonance states, J. Phys. B: At. Mol. Phys. 15 (1982), 1-15.

[18] Schuch D., Relations between wave and particle aspects for motion in a magnetic field, in New Challenges in Computational Quantum Chemistry, Editors R. Broer, P.J.C. Aerts and P.S. Bagus, University of Groningen, 1994, 255-269.

Maamache M. Bounames A., Ferkous N., Comment on "Wave function of a time-dependent harmonic oscillator in a static magnetic field", Phys. Rev. A 73 (2006), 016101, 3 pages.

[19] Ray J.R., Time-dependent invariants with applications in physics, Lett. Nuovo Cim. 27 (1980), 424-428. Sarlet W., Class of Hamiltonians with one degree-of-freedom allowing applications of Kruskal's asymptotic theory in closed form. II, Ann. Phys. (N.Y.) 92 (1975), 248-261.

[20] Lewis H.R., Leach P.G.L., Exact invariants for a class of time-dependent nonlinear Hamiltonian systems, J. Math. Phys. 23 (1982), 165-175.

[21] Sebawa Abdalla M., Leach P.G.L., Linear and quadratic invariants for the transformed Tavis-Cummings model, J. Phys. A: Math. Gen. 36 (2003), 12205-12221.

Sebawa Abdalla M., Leach P.G.L., Wigner functions for time-dependent coupled linear oscillators via linear and quadratic invariant processes, J. Phys. A: Math. Gen. 38 (2005), 881-893.

[22] Kaushal R.S., Classical and quantum mechanics of noncentral potentials. A survey of 2D systems, Springer, Heidelberg, 1998. 